\title{
BMJ Open Comparison of the ultra-low-dose Veo algorithm with the gold standard filtered back projection for detecting pulmonary asbestos-related conditions: a clinical observational study
}

\author{
Marielle Tekath, ${ }^{1}$ Frédéric Dutheil, ${ }^{2,3,4,5}$ Romain Bellini, ${ }^{6}$ Antoine Roche, ${ }^{1}$ \\ Bruno Pereira, ${ }^{7}$ Geraldine Naughton, ${ }^{3}$ Alain Chamoux, ${ }^{2}$ Jean-Luc Michel ${ }^{1}$
}

To cite: Tekath M, Dutheil F, Bellini R, et al. Comparison of the ultra-low-dose Veo algorithm with the gold standard filtered back projection for detecting pulmonary asbestos-related conditions: a clinical observational study. BMJ Open 2014;4:e004980. doi:10.1136/bmjopen-2014004980

- Prepublication history for this paper is available online. To view these files please visit the journal online (http://dx.doi.org/10.1136/ bmjopen-2014-004980).

Received 1 February 2014 Revised 30 April 2014 Accepted 7 May 2014

CrossMark

For numbered affiliations see end of article.

Correspondance to Geraldine Naughton; Geraldine.Naughton@acu. edu.au

\section{ABSTRACT}

Objectives: Radiation delivered during CT is a major concern, especially for individuals undergoing repeated screening. We aimed to compare a new ultra-low-dose algorithm called Veo with the gold standard filtered back projection (FBP) for detecting pulmonary asbestos-related conditions.

Setting: University Hospital CHU G. Montpied, Clermont-Ferrand, France

Participants: Asbestos-exposed workers were recruited following referral to screening for asbestosrelated conditions. Two acquisitions were performed on a 64-slice CT: the gold standard FBP followed by Veo reconstruction.

Outcome measures: Two radiologists independently assessed asbestos-related abnormalities, pulmonary nodules, radiation doses and image quality (noise).

Results: We included 27 asbestos-exposed workers $(63.3 \pm 6.5$ years with $11.9 \pm 9.7$ years of asbestos exposure). We observed 297 pleural plaques in 20 participants $(74 \%)$. All patients $(100 \%)$ had pulmonary nodules, totalling 167 nodules. Detection rates did not differ for pleural plaques (Veo $87 \%$ vs FBP $97 \%$, NS), pleural thickening (100\% for both) and pulmonary nodules ( $80 \%$ for both). Interstitial abnormalities were depicted less frequently with Veo than FBP. False negative and false positive did not exceed $2.7 \%$. Compared with FBP, Veo decreased the radiation dose up to $87 \%$ (Veo $0.23 \pm 0.07$ vs FBP $1.83 \pm 0.88 \mathrm{mSv}$, $\mathrm{p}<0.001)$. The objective image noise also decreased with $\mathrm{VeO}$ as much as $23 \%$ and signal-to-noise ratio increased up to $33 \%$.

Conclusions: A low-dose CT with Veo reconstruction substantially reduced radiation. Veo compared favourably with FBP in detecting pleural plaques, pleural thickening and pulmonary nodules. These results should be confirmed on a larger sample size before the use of Veo in clinical routine practice in asbestos-related conditions, especially regarding the low prevalence of interstitial abnormalities in this study.

Trial registration number: NCT01955018.
Strengths and limitations of this study

- Radiation delivered during CT is a major concern, especially for individuals undergoing repeated screening, such as asbestos-exposed workers.

- We provide the first comparison of a new ultra-low-dose algorithm called Veo ('I see' in Spanish) with the gold standard filtered back projection (FBP) in detecting pulmonary conditions in asbestos-exposed workers.

- Veo substantially reduces radiation doses, with $87 \%$ less radiation delivered than FBP.

- Veo compared favourably with FBP acquisitions in detecting pleural plaques, diffuse pleural thickening and pulmonary nodules; the high prevalence of pleural plaques (297, observed in $74 \%$ of participants) and pulmonary nodules (167) permitted a robust statistical analysis.

- However, these results should be confirmed on a larger sample size before the use of Veo in clinical routine practice in asbestos-related conditions, especially regarding the low prevalence of interstitial abnormalities.

\section{INTRODUCTION}

Asbestos fibres were intensively used throughout the 20th century, and remain prevalent in developing countries. ${ }^{1}$ However, asbestos exposure induces a variety of benign and malignant pleural and lung diseases. ${ }^{2} 3$ Owing to a long latency period between exposure and disease presentation, asbestosrelated diseases remain a substantial public health problem. ${ }^{1}$ The most common asbestos-induced neoplasm is lung cancer. ${ }^{2} 3$ Chest CT screening has been successfully used in the early detection of lung cancer in asbestos-exposed workers. ${ }^{4-6}$ Moreover, thinsection CT is more sensitive than a chest X-ray for detecting early asbestos-related 
conditions. ${ }^{7-10}$ Nevertheless, the use of CT has two main disadvantages: high radiation doses and depiction of incidental abnormalities such as pulmonary nodules in asymptomatic patients. Incidental abnormalities increase the frequency of follow-up by CT and may also psychologically impact on patients. Medical exposure from $\mathrm{X}$-rays represents the major source of man-made irradiation with a large contribution from CT. ${ }^{11-13}$ Increased exposure to radiation underpins the consequences of cancer induction. ${ }^{14}$ However, reducing CT doses increases image noise from the filtered back projection (FBP) reconstruction. Strategies to reduce radiation exposure include the use of iterative reconstruction algorithms such as 'iDose', '100\% ASIR (adaptive statistical iterative reconstruction)' and 'IRIS (iterative reconstruction in image space) ${ }^{\prime} \cdot{ }^{15-20}$ The new algorithm called Veo (General Electric Healthcare, Milwaukee, Wisconsin, USA) decreases the image noise up to $70 \%$ compared with the gold standard FBP model, whereas the '100\% ASIR' algorithm is only capable of reducing image noise up to $47 \% .{ }^{21}$ Moreover, Veo ('I see' in Spanish) improves spatial resolution with excellent detection of low-contrast and high-contrast objects from a CT Dose Index $\left(\mathrm{CTDI}_{\mathrm{vol}}\right)$ equal to $0.3 \mathrm{mGy}$.

Thus, the objective of the present study was to compare Veo with the gold standard FBP for detecting pulmonary asbestos-related conditions among workers previously exposed to asbestos. Comparisons included radiation delivered and image quality.

\section{METHODS}

\section{Patients}

Written informed consent was obtained from all participants for the supplementary acquisition of Veo images in addition to their clinically indicated chest CT. Asbestos-exposed workers were recruited following referral to our radiology department for the evaluation of asbestos-related disease between September 2012 and April 2013. Inclusion criteria were: being asbestosexposed workers, having a chest CT referral from the occupational medicine department, no history of cancer or thoracic surgery and the absence of other known interstitial pathology.

\section{CT protocol}

CT examinations were performed with a 64-slice CT system (Discovery CT 750HD; GE Healthcare, Milwaukee, Wisconsin, USA) and consisted of two successive acquisitions. Each examination, the normal-dose (FBP acquisition) and ultra-low-dose (Veo acquisition) spiral CT, was obtained on the entire thorax, at full inspiration with the participant in the supine position. No intravenous contrast material was administered. In accordance with guidelines, standard acquisition was performed with CT parameters adjusted to the participant's body size, including a tube kilovoltage $(\mathrm{kV})$ of 120 (participants weighing $70 \mathrm{~kg}$ or less) and 140 (participants weighing more than
$70 \mathrm{~kg})$, with milliamperage $(\mathrm{mA})$ equal to the patient's body weight. The other CT parameters were rotation time $0.5 \mathrm{~s}$ and pitch 1.375. Image data were reconstructed with FBP algorithm. The Veo acquisition was performed with constant CT parameters including: a tube voltage of $100 \mathrm{kV}$, a tube current of $20 \mathrm{~mA}$, pitch of 0.984 and rotation time of $0.4 \mathrm{~s}$. Image data were reconstructed with the Veo algorithm.

\section{Interpretation of CT images}

Each CT acquisition was viewed independently by two radiologists (2 and 7 years of experience- $\mathrm{RB}$ and $\mathrm{AR}$ ). The low-dose images with Veo reconstruction were interpreted before the standard CT and on separate weeks to minimise recall bias. A third simultaneous reading of the Veo and FBP acquisitions by the more experienced radiologist (AR) evaluated the concordance of pleuroparenchymal abnormalities between $V e o$ and FBP. Because FBP images are benchmark practice, when a lesion was found only on Veo images, it was regarded as a false positive.

\section{Pleural and parenchymal abnormalities}

According to established criteria, ${ }^{9}{ }^{22} 23$ the following asbestos-related pleural and parenchymal abnormalities were recorded as present or absent. Pleural abnormalities considered were:

- Pleural plaques: pleura thickening with no associated parenchymal abnormality. We recorded for each lesion: localisation (side, region: anterolateral, posterolateral, diaphragmatic or mediastinum), thickness and calcification.

- Diffuse pleural thickening: pleural thickening associated with parenchymal abnormalities such as rounded atelectasis and parenchymal bands. ${ }^{22}$

- Pleural effusion is typically asymptomatic, the fluid may be serous or haemorrhagic. ${ }^{22}$

CT features of asbestosis included: (1) subpleural dots and branching opacities, (2) curvilinear subpleural lines, defined as linear opacity within $1 \mathrm{~cm}$ of the pleura and parallel to the inner chest wall, (3) areas of ground glass opacities, (4) septal lines and (5) reticulations defined as single or branching lines $1-2 \mathrm{~cm}$ in length in the subpleural parenchyma, and (6) honeycombing, defined as cystic air spaces with well-defined walls less than $1 \mathrm{~cm}$ in diameter.

Presence of nodules was also recorded. We noted for each abnormality: localisation (side, table position) and nature (non-solid, part-solid, solid or calcified). To increase sensitivity, nodules were examined by combining maximum intensity projections and millimetric axial CT images. ${ }^{24}$

\section{Radiation}

Comparisons included the dose length product (DLP) in $\mathrm{mGy} \mathrm{cm}$ and effective doses in mSv. Computed conversion factor from DLP to effective dose for adult chest is $0.0146 \mathrm{mSv} / \mathrm{mGy} / \mathrm{cm}^{25}$ 
Quality of FBP and Veo images

Respiratory artefacts were graded on a three-point scale ( $1=$ negligible, $2=$ moderate, $3=$ salient $)$. Image noise was studied in the axial and coronal planes. A similar scale was used for subjective image quality in the mediastinum and parenchyma windows. Objective image noise is the mean of the SD of the signal intensity (in Hounsfield's units) measured with circular regions of interest (ROI) on different anatomical levels, $10 \mathrm{~mm}$ in diameter. ${ }^{19}$ ROIs were drawn within the descending thoracic aorta at the level of the left main bronchus, within the tracheal lumen up to the tracheal bifurcation, and on the lung. The signal-to-noise ratio (SNR) was also calculated using the equation $\mathrm{SNR}=$ signal intensity/objective noise. ${ }^{26}$

\section{Statistical analysis}

Sample size estimation was based on the number of pleural plaques and nodules. Considering the investigative nature of the study design and because the number of pleural plaques and nodules was not known initially, a sample size estimation was not proposed a priori even though a concordance coefficient $\kappa$ between 0.40 and 0.90 was expected between FBP and Veo images. Therefore, 30 asbestos-exposed workers were predicted to be necessary to reject the null hypothesis ' $\mathrm{H} 0$ : $\kappa=0.40$ ' vs 'H1: $\kappa \neq 0.75$ ' for a proportion of pleural plaques of $65 \%$, with a statistical power of $>85 \%$ and $\alpha=5 \%$ (twosided). Finally, the study was conducted to sequentially control the statistical power considering the number of plaques and nodules for each asbestos-exposed worker. ${ }^{27}$

Statistical analysis was performed using Stata software (V.12; Stata-Corp, College Station, Texas, USA). Quantitative variables are expressed as means \pm SDs. Proportions are expressed as percentage and 95\% CIs. Comparisons in paired situation were realised using paired Student $\mathrm{t}$ test or Wilcoxon test when appropriate for quantitative variables and Stuart-Maxwell test for categorical parameters. Sensitivity, specificity, false positives and false negatives values of Veo were calculated and presented with $95 \%$ CIs, in comparison with results from FBP acquisitions. Estimates of sensitivity and specificity were obtained by first estimating the sensitivity and specificity for each patient. Sensitivity and specificity were then estimated by averaging the individual-specific estimates across patients. The variance of the estimate was the sample variance divided by the number of patients. Generalised estimating equation models with logit link and working independence correlation structure were also used to estimate sensitivity, taking into account the correlation among the multiple pleural plaques and pulmonary nodules for the same patient. The $\kappa$ coefficient was used to measure agreement for categorical parameters and Pearson's correlation coefficient and Lin concordance correlation coefficient for quantitative data. The analyses were completed by using random-effect models when appropriate to consider within and between participant variability. The tests were two-sided, with a type I error set at $\alpha=0.05$.

\section{RESULTS}

\section{Patients}

The flow chart of participants is displayed in figure 1 . Among the 87 asbestos-exposed workers referred to our radiology department, 29 gave their consent and 27 were retained for analyses. The mean age of volunteers was $63.3 \pm 6.5$ years. The mean duration of occupational exposure was $11.9 \pm 9.7$ years.

\section{Radiation dose}

The average DLP was $16 \pm 5 \mathrm{mGy} \mathrm{cm}$ for $V e o$ and $125 \pm 61$ $\mathrm{mGy} \mathrm{cm}$ for FBP. The corresponding average effective doses were $0.234 \pm 0.073 \mathrm{mSv}$ for $\mathrm{Veo}$ and $1.825 \pm 0.876 \mathrm{mSv}$ for FBP. The dose reduction was calculated to be $87.2 \%$ $(\mathrm{p}<0.001)$.

\section{Quality images assessment}

For Veo acquisition, respiratory artefacts were graded as 'negligible' in 24 cases (89\%) for reader 1 and 25 cases $(93 \%)$ for reader 2 , 'moderate' in 3 cases $(11 \%)$ for reader 1 and 2 cases $(7 \%)$ for reader 2 , and no 'salient' artefact was recorded. For FBP acquisition, respiratory artefacts were graded as 'negligible' in 19 cases $(70 \%)$ for reader 1 and 24 cases (89\%) for reader 2, 'moderate' in 7 cases $(26 \%)$ for reader 1 and 3 cases (11\%) for reader 2 , and as 'salient' in 1 case for reader 1 and in 0 cases for

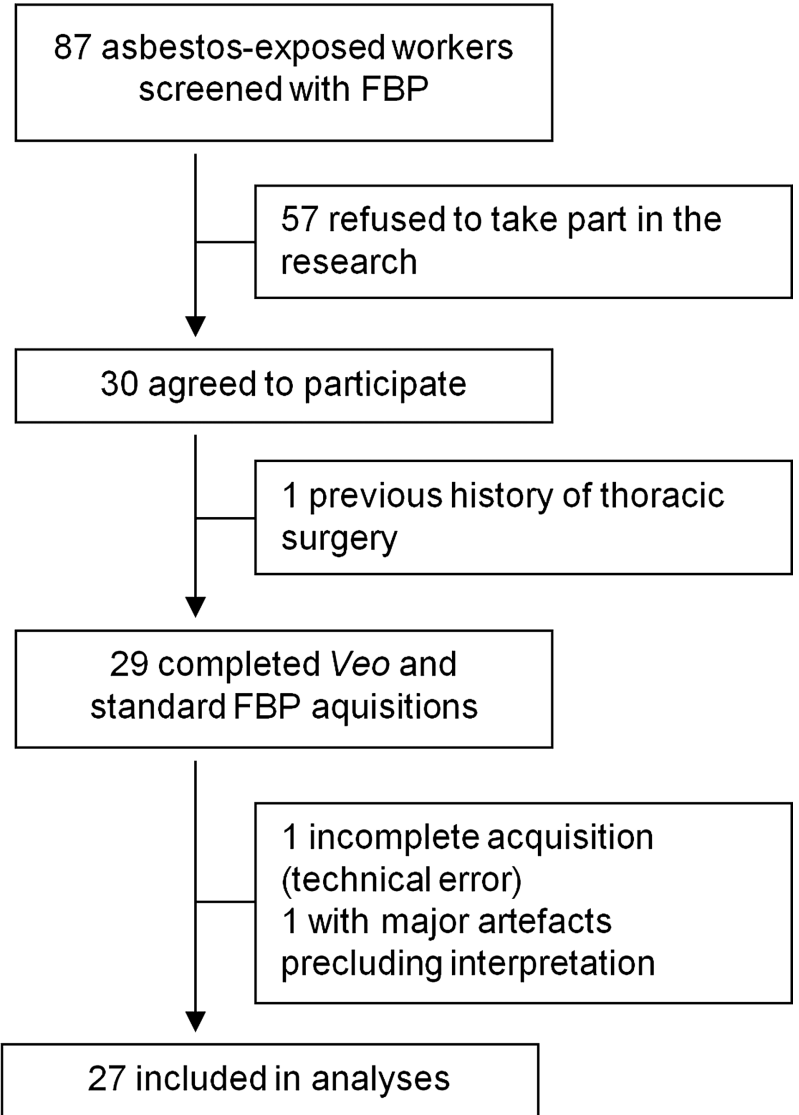

Figure 1 Flow chart of participants. FBP, filtered back projection. 
Table 1 Subjective noise assessment

\begin{tabular}{|c|c|c|c|c|c|c|c|c|}
\hline & \multicolumn{2}{|c|}{ Axial mediastinum } & \multicolumn{2}{|c|}{ Axial parenchyma } & \multicolumn{2}{|c|}{$\begin{array}{l}\text { Coronal } \\
\text { mediastinum }\end{array}$} & \multicolumn{2}{|c|}{$\begin{array}{l}\text { Coronal } \\
\text { parenchyma }\end{array}$} \\
\hline & Veo & FBP & Veo & FBP & Veo & FBP & Veo & FBP \\
\hline \multicolumn{9}{|l|}{ Reader 1} \\
\hline Minimal, no (\%) & $2(7)$ & $22(82)$ & $6(21)$ & $25(93)$ & $19(68)$ & $25(93)$ & $22(79)$ & $26(96)$ \\
\hline Moderate, no (\%) & $19(68)$ & $5(18)$ & $18(64)$ & $2(7)$ & $8(32)$ & $2(7)$ & $5(21)$ & $1(4)$ \\
\hline Important, no (\%) & $6(25)$ & 0 & $3(14)$ & 0 & 0 & 0 & 0 & 0 \\
\hline p Value & $<0.001$ & & $<0.001$ & & 0.03 & & 0.10 & \\
\hline \multicolumn{9}{|l|}{ Reader 2} \\
\hline Minimal, no (\%) & $2(7)$ & $17(64)$ & $4(14)$ & $26(96)$ & $8(29)$ & $19(68)$ & $19(68)$ & $26(96)$ \\
\hline Moderate, no (\%) & $14(50)$ & $10(36)$ & $18(64)$ & $1(4)$ & $17(61)$ & 7 (29) & $8(32)$ & $1(4)$ \\
\hline Important, no (\%) & $11(43)$ & 0 & $5(21)$ & 0 & $2(11)$ & $1(4)$ & 0 & 0 \\
\hline p Value & $<0.001$ & & $<0.001$ & & 0.02 & & $<0.01$ & \\
\hline
\end{tabular}

FBP, filtered back projection.

reader 2. Veo and FBP did not differ in subjective assessment of respiratory artefacts between the two radiologists $(\mathrm{p}=0.16$ for reader 1 and $\mathrm{p}=0.65$ for reader 2$)$.

Tables 1 and 2 provide results from subjective image noise assessed by the two radiologists using, average of objective noise data and SNR. The two protocols differed significantly in objective image noise. The ultra-low-dose Veo acquisition reduced objective image noise from $13 \%$ to $23 \%$ and increased SNR from $5 \%$ to $33 \%$ compared with the standard FBP acquisition.

However, the two readers rated higher subjective image noise in axial and coronal planes with Veo than FBP, with the exception of parenchymal analysis in the coronal plane for the reader 1 (table 1$)$.

\section{Pleural plaques}

A total of 297 pleural plaques (figure 2) were observed in 20 participants $(74 \%)$. Detection of plaques did not differ between Veo $(259 ; 87 \%)$ and FBP (287; 97\%; $\mathrm{p}=0.10)$. Thus, the third simultaneous reading of Veo and FBP resulted in the detection of 10 plaques that were not detected during the first reading of FBP images. The agreement for pleural plaques depiction was $84 \%$ with a $\kappa$ of 0.05 . However, when data were examined only for the presence of pleural plaque (yes or no) in patients, agreement increased to $96 \%$ $(\kappa=0.91) .^{28}{ }^{29}$ Moreover agreement for size measurement (Lin coefficient) was $0.83(\mathrm{p}<0.001)$ and $\kappa$ coefficient for calcification detection was 0.86 .

For one participant, despite a Veo acquisition considered normal, FBP acquisition was positive for one isolated plaque.

Simultaneous analysis of Veo and FBP acquisitions led us to observe that Veo acquisition was responsible for three false positives corresponding to intercostal fat or muscles, with eight false negatives $(2.7 \%)$.

\section{Pleural thickening}

Diffuse pleural thickening (figure 2) was present in four patients $(14.8 \%)$. The detection rate for each technique was $100 \%$ with a $\kappa$ of 1 . No pleural effusion was found.

\section{Parenchymal abnormalities}

Parenchymal changes were found in 15 participants (55.6\%), including subpleural dots, curvilinear subpleural lines, ground glass opacities, septal lines and reticulations. No honeycombing was found. Table 3 summarises the prevalence $(\mathrm{P})$, inter-rater agreement $(\tau)$

Table 2 Objective noise and signal-to-noise ratio measurements

\begin{tabular}{|c|c|c|c|c|c|c|c|c|}
\hline & \multicolumn{4}{|c|}{ Objective noise } & \multicolumn{4}{|c|}{ signal-to-noise ratio } \\
\hline & $\begin{array}{l}\text { Veo } \\
\text { Mean士SD }\end{array}$ & $\begin{array}{l}\text { FBP } \\
\text { Mean+SD }\end{array}$ & p Value & Decrease (\%) & $\begin{array}{l}\text { Veo } \\
\text { Mean士SD }\end{array}$ & $\begin{array}{l}\text { FBP } \\
\text { Mean } \pm S D\end{array}$ & p Value & Increase (\%) \\
\hline \multicolumn{9}{|l|}{ Trachea } \\
\hline Axial & $20.1 \pm 3.6$ & $26.1 \pm 9.3$ & $<0.01$ & -23 & $47.5 \pm 7.8$ & $42.5 \pm 10.9$ & 0.04 & 12 \\
\hline Coronal & $21.1 \pm 3.4$ & $24.4 \pm 7.1$ & 0.02 & -13 & $45.7 \pm 7.3$ & $43.4 \pm 9.7$ & 0.24 & 5 \\
\hline \multicolumn{9}{|c|}{ Descending aorta } \\
\hline Axial & $20.1 \pm 3.2$ & $25.1 \pm 5.4$ & $<0.001$ & -20 & $1.7 \pm 0.6$ & $1.3 \pm 0.3$ & $<0.001$ & 33 \\
\hline Coronal & $19.8 \pm 3.3$ & $24.5 \pm 4.5$ & $<0.001$ & -19 & $1.6 \pm 0.6$ & $1.3 \pm 0.3$ & $<0.01$ & 22 \\
\hline \multicolumn{9}{|l|}{ Lung } \\
\hline Axial & $25.8 \pm 5.1$ & $32.8 \pm 14.4$ & 0.02 & -21 & $34.1 \pm 6.6$ & $31.6 \pm 10.5$ & 0.25 & 8 \\
\hline Coronal & $27.2 \pm 6.1$ & $34.3 \pm 10.5$ & $<0.001$ & -21 & $32.3 \pm 7.3$ & $28.5 \pm 6.9$ & 0.03 & 13 \\
\hline
\end{tabular}


Figure 2 Typical pleural plaques (1; white arrows), diffuse pleural thickening (2; white arrows) and parenchymal band (2; black arrows), and pulmonary nodule (3; white arrows) in axial plane and an example of normal images in axial plane (4). All Veo and filtered back projection (FBP) images are captured at the same anatomic level, with $100 \mathrm{kV}$ and $20 \mathrm{mAs} /$ section for $\mathrm{Veo}$ and $120 \mathrm{kV}, 60 \mathrm{mAs}$ for FBP.

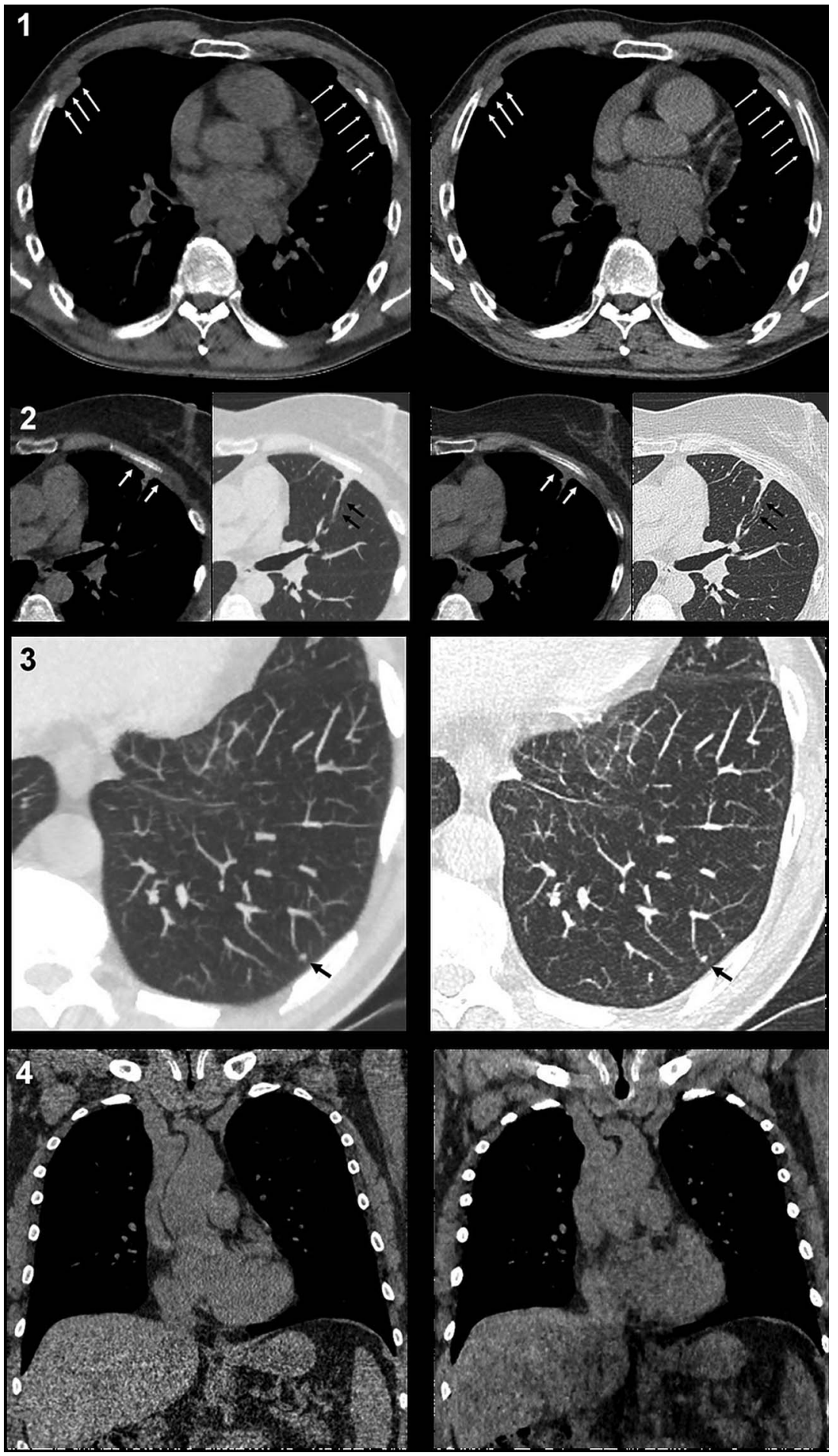

and $\kappa$ between Veo and FBP acquisition, and the sensitivity, specificity, predictive positive value and predictive negative value of $V e o$ acquisition for each interstitial abnormality.

\section{Pulmonary nodules}

Pulmonary nodules (figure 2) were found in all patients, with a total of 167 nodules. All the nodules detected were smaller than $10 \mathrm{~mm}$. No non-solid or part-solid nodules were observed. Among the 167 recorded nodules, the detection rate did not differ $(\mathrm{p}=0.98)$ between Veo (134/ $167)$ and standard FBP $(133 / 167)$, with the same $80 \%$ detection rate. Thus, the third simultaneous reading of $V e o$ and FBP resulted in the detection of 34 nodules that were not detected during the first reading of FBP images. The agreement between the two techniques for nodules depiction was $60 \%(\kappa=0.25)$. Simultaneous analysis of Veo and FBP acquisitions permitted us to observe that Veo acquisition was responsible for seven false positives $(4 \%)$ and four false negatives $(2.7 \%)$. 
Table 3 Low-dose CT scan with Veo reconstruction, accuracy for interstitials abnormalities

\begin{tabular}{|c|c|c|c|c|c|c|c|}
\hline & $\mathbf{P}(\%)$ & $\tau(\%)$ & к & $\mathrm{Se}(\%, \mathrm{Cl} 95 \%)$ & $\mathrm{Sp}(\%, \mathrm{Cl} 95 \%)$ & PPV (\%, CI 95\%) & PNV (\%, CI 95\%) \\
\hline Total & 55.6 & 70.4 & 0.44 & 46.7 (21.3 to 73.4$)$ & 100 (73.5 to 100$)$ & 100 (59 to 100$)$ & 60 (36.1 to 80.9$)$ \\
\hline $\begin{array}{l}\text { Subpleural dots and } \\
\text { branching opacities }\end{array}$ & 33.3 & 74 & 0.34 & 33.3 (7.5 to 70.1$)$ & 94.4 (72.7 to 99.9$)$ & 75 (19.4 to 99.4$)$ & 73.9 (51.6 to 89.8$)$ \\
\hline Curvilinear subpleural lines & 8 & 0 & 0 & 0 & 0 & 0 & 0 \\
\hline $\begin{array}{l}\text { Areas of ground glass } \\
\text { opacities }\end{array}$ & 25.9 & 77.8 & 0.2 & $14.3(0.4$ to 57.9$)$ & 100 (83.2 to 100$)$ & $100(2.5$ to 100$)$ & 76.9 (56.4 to 91$)$ \\
\hline Honeycombing & 0 & & & & & & \\
\hline Reticulations & 18.5 & 92.6 & 0.71 & $60(14.7$ to 94.7$)$ & $100(84.6$ to 100$)$ & 100 (29.2 to 100$)$ & 91.7 (73 to 99$)$ \\
\hline Septal lines & 14.8 & 85.2 & 0.26 & 25 (0.6 to 80.6$)$ & 95.7 (78.1 to 99.9$)$ & $50(1.3$ to 98.7$)$ & 88 (68.8 to 97.5$)$ \\
\hline
\end{tabular}

\section{Interobserver agreement}

No difference was observed for the interobserver agreement $(\kappa)$ between the two techniques. Interobserver agreement was low for pleural plaques detection (0.09 for FBP and 0.10 for $V e o$ ) and fair for nodule detection ( 0.34 for FBP and 0.34 for Veo). The inter-reader agreements for parenchymal interstitial abnormalities and parenchymal diffuse pleural thickening were not evaluated due to their low prevalence.

\section{DISCUSSION}

We compared for the first time low-dose CT using Veo reconstruction and the gold standard CT using FBP reconstruction to depict asbestos-related abnormalities and pulmonary nodules depiction. The major finding was that Veo compared favourably with FBP acquisitions in detecting pleural plaques, diffuse pleural thickening and pulmonary nodules. However, interstitial parenchymal abnormalities were depicted less frequently in Veo than FBP acquisitions. Nevertheless, Veo delivered $87 \%$ less radiation than FBP.

\section{Quality of images}

The assessment of image quality showed discordant results. Despite low scanning parameters, the iterative reconstruction method of Veo significantly reduced the level of objective noise, but subjective noise parameters increased in comparison with FBP. This discordance may be explained by the novel appearance of Veo images requiring adaptation time for the radiologists. Our results are in line with previous results showing a relative noise reduction of $25 \%$ obtained from Veo $(100 \mathrm{kV}$, $10 \mathrm{mAs})$ compared with FBP protocol $(100 \mathrm{kV}, 50$ $300 \mathrm{mAs}){ }^{30}$

\section{Pleural plaques}

Pleural plaques corresponding to parietal pleura fibrosis are indicators of asbestos exposure ${ }^{7}$ with a prevalence as high as $60 \%$ in previously exposed workers ${ }^{10} 31$ and $74 \%$ in the current study with highly exposed workers. In France, the detection of pleural plaques results in financial compensation for workers and early retirement.
Consequently, pleural plaques are accepted only when results are unequivocal. Atypical plaques will only be considered when they occur bilaterally or in multiple sites, and with typical localisation. Owing to the three cases of false positive and eight cases of false negative, the low-dose CT with Veo reconstruction cannot be used for the first examination, but its use seems possible for patients' follow-up.

\section{Diffuse pleural thickening detection}

Our results indicated that a low-dose scanner with Veo reconstruction was comparable with the FBP gold standard for diffuse pleural thickening detection. The prevalence of thickening was rare thus, we could not obtain statistical significance. However, thickening is of major importance because diagnosis results not only in compensation, but guarantees a lifelong pension. Considering the importance usually noted about these lesions, a Veo acquisition should be sufficient in clinical practice.

\section{Parenchymal abnormalities}

Reader sensitivity with Veo images was poor for interstitial parenchymal abnormalities. No case of true asbestosis was recorded, but 15 patients had non-specific interstitial abnormalities. However, the detection of interstitial abnormalities may be limited by several factors. First, the study was built for asbestos-related diseases. Recording specifications lacked the details required to comprehensively describe the presence of interstitial abnormalities. Therefore, without systematic records, interstitial abnormalities were likely underestimated. Second, Veo was always performed after FBP resulting in an increase of gravity-dependent attenuation in the posterior region which may have masked interstitial abnormalities. Third, the acquisitions were performed in the supine position, and acquisitions in the prone position were not always performed when necessary. Subsequently, the posterior region was not analysed with confidence. Thus, interstitial abnormalities were underestimated in our study.

Asbestosis refers to interstitial fibrosis caused by the deposition of asbestos fibres in the lung. Its prevalence is 
estimated to be about $5 \%$ in asbestos-exposed workers. ${ }^{32}$ Asbestosis remains difficult to diagnose, particularly in the early stages. However, a significant dose-effect relationship exists between the cumulative exposure to asbestos and asbestosis. ${ }^{33}$ Asbestosis is usually associated with dyspnoea, basilar rales and changes in pulmonary function with restrictive or mixed restrictive-obstructive patterns, and carbon monoxide diffusion abnormalities. Pulmonary asbestosis was previously diagnosed in 51 of $706(7 \%)$ asbestos-exposed workers. ${ }^{34}$ In a previous study, 51 of the $706(7 \%)$ asbestos-exposed workers were diagnosed with pulmonary asbestosis. In this study, only $2 \%$ of the workers with less than 25 years of cumulative exposure to asbestos were diagnosed with asbestosis using high resolution CT screening. ${ }^{34}$ Therefore, CT screening for asbestosis does not seem warranted in workers with low occupational exposure.

\section{Pulmonary nodules}

In our study, all individuals had at least one pulmonary nodule. FBP and Veo shared the same detection rate of $80 \%$. However, Veo reconstruction is not advised for initial nodules screening due to the seven false positives and four false negatives from the 167 nodules. According to the Fleischner Society guidelines, ${ }^{35}$ nodule detection on CT requires specific management. In agreement with our recommendations for pleural plaques detection, Veo should be used only for patients' follow-up after a first detection of pulmonary nodules using gold standard CT.

\section{Comparison of Veo with other low-dose algorithms}

To date, no study using other algorithms to reduce radiation exposure has investigated asbestos-related conditions. Thus, because the Veo algorithm appears to reduce the more radiation delivered than other low-dose algorithms such as 'iDose', '100\% ASIR' or 'IRIS', ${ }^{15-20}$ we chose only to compare Veo with the gold standard FBP.

\section{Limitations}

The sample size could be perceived as a limitation. Limited sample size exacerbated the need for rapid adaptation time by the radiologists with relatively novel images. However, statistically, the high prevalence of pleural plaques (297, observed in $74 \%$ of participants) and pulmonary nodules (167) permitted a robust statistical analysis. Considering these results $(\kappa=0.91)$, power seemed satisfactory $(80 \%)$ to reject the null hypothesis ' $\mathrm{H} 0: \kappa=0.4$ ' vs ' $\mathrm{H} 1: \kappa \neq 0.91$ ' with 27 patients. In contrast, parenchymal interstitial abnormalities were rare, precluding sound statistical analyses. Parenchymal interstitial abnormalities suffered from major limitations due to CT positioning of patients. A further study dedicated to parenchymal interstitial abnormalities should be conducted. Clinically, a current limitation of iterative reconstruction is a long computing time.

\section{CONCLUSION}

A low-dose CT with Veo reconstruction substantially reduces radiation. Despite an unusual appearance, Veo image quality was generally accurate in its diagnosis. Specifically, Veo compared favourably with the gold standard FBP acquisitions in detecting pleural plaques, diffuse pleural thickening and pulmonary nodules. However, these results should be confirmed on a larger sample size before the use of Veo in clinical routine practice in asbestos-related conditions, especially regarding the low prevalence of interstitial abnormalities in this study.

\section{Author affiliations}

${ }^{1}$ Department of Radiology, University Hospital CHU G. Montpied, ClermontFerrand, France

${ }^{2}$ Department of Occupational Medicine, University Hospital CHU G. Montpied, Clermont-Ferrand, France

${ }^{3}$ School of Exercise Science, Australian Catholic University, Fitzroy, Victoria, Australia

${ }^{4}$ Laboratory of Metabolic Adaptations to Exercise in Physiological and Pathological Conditions EA3533, Blaise Pascal University, Clermont-Ferrand, France

${ }^{5}$ INRA, UMR 1019, UNH, CRNH Auvergne, Clermont-Ferrand, France ${ }^{6}$ Department of Radiology, Centre Jean Perrin, University Hospital CHU, Clermont-Ferrand, France

${ }^{7}$ Department of Medical Statistics, University Hospital CHU G. Montpied, Clermont-Ferrand, France

Acknowledgements The authors would like to thank the 'CAPER des Combrailles' (Association of the asbestos-exposed workers), which helped to recruit the asbestos-exposed workers.

Contributors MT, FD, AC and J-LM generated the intellectual development of the study. MT and FD obtained the ethics approval. FD and AC recruited all participants. RB and AR completed the double blind analyses of $V e o$ and FBP images. MT and BP made data analysis. MT drafted the manuscript. FD and GN revised the manuscript. All authors read and approved the final manuscript.

Funding The study was funded by the University Hospital CHU G. Montpied, 58 rue Montalembert 63000 Clermont-Ferrand, France.

Competing interests MT, FD, AC and J-LM obtained research funding.

Patient consent Obtained.

Ethics approval Approval was given by the ethics committee of the University Hospital of Clermont-Ferrand (ClinicalTrials.gov number: NCT01955018).

Provenance and peer review Not commissioned; externally peer reviewed.

Data sharing statement Original data (crude data) from this study are available by contacting the corresponding author via email.

Open Access This is an Open Access article distributed in accordance with the Creative Commons Attribution Non Commercial (CC BY-NC 3.0) license, which permits others to distribute, remix, adapt, build upon this work noncommercially, and license their derivative works on different terms, provided the original work is properly cited and the use is non-commercial. See: http:// creativecommons.org/licenses/by-nc/3.0/

\section{REFERENCES}

1. Stayner L, Welch LS, Lemen R. The worldwide pandemic of asbestos-related diseases. Ann Rev Public Health 2013;34:205-16.

2. Oksa P, Pukkala E, Karjalainen A, et al. Cancer incidence and mortality among Finnish asbestos sprayers and in asbestosis and silicosis patients. Am J Ind Med 1997;31:693-8.

3. Koskinen K, Pukkala E, Martikainen R, et al. Different measures of asbestos exposure in estimating risk of lung cancer and mesothelioma among construction workers. J Occup Environ Med 2002;44:1190-6. 
4. Clin B, Morlais F, Guittet L, et al. Performance of chest radiograph and CT scan for lung cancer screening in asbestos-exposed workers. Occup Environ Med 2009;66:529-34.

5. Fasola G, Belvedere O, Aita M, et al. Low-dose computed tomography screening for lung cancer and pleural mesothelioma in an asbestos-exposed population: baseline results of a prospective, nonrandomized feasibility trial—an Alpe-adria Thoracic Oncology Multidisciplinary Group Study (ATOM 002). Oncologist 2007;12:1215-24.

6. Vierikko T, Jarvenpaa R, Autti T, et al. Chest CT screening of asbestos-exposed workers: lung lesions and incidental findings. Eur Respir J 2007;29:78-84.

7. American Thoracic Society. Diagnosis and initial management of nonmalignant diseases related to asbestos. Am J Respir Crit Care Med 2004;170:691-715.

8. Akira M, Yamamoto S, Yokoyama K, et al. Asbestosis: highresolution CT-pathologic correlation. Radiology 1990;176:389-94.

9. Akira M, Yokoyama K, Yamamoto S, et al. Early asbestosis: evaluation with high-resolution CT. Radiology 1991;178:409-16.

10. Neri S, Antonelli A, Falaschi F, et al. Findings from high resolution computed tomography of the lung and pleura of symptom free workers exposed to amosite who had normal chest radiographs and pulmonary function tests. Occup Environ Med 1994;51:239-43.

11. Mettler FA Jr, Thomadsen BR, Bhargavan M, et al. Medical radiation exposure in the U.S. in 2006: preliminary results. Health Phys 2008;95:502-7.

12. Samara ET, Aroua A, Bochud FO, et al. Exposure of the Swiss population by medical x-rays: 2008 review. Health Phys 2012;102:263-70.

13. Shrimpton PC, Hillier MC, Lewis MA, et al. National survey of doses from CT in the UK: 2003. Br J Radiol 2006;79:968-80.

14. Brenner DJ, Hall EJ. Computed tomography-an increasing source of radiation exposure. N Engl J Med 2007;357:2277-84.

15. Marin D, Nelson RC, Schindera ST, et al. Low-tube-voltage, high-tube-current multidetector abdominal CT: improved image quality and decreased radiation dose with adaptive statistical iterative reconstruction algorithm-initial clinical experience. Radiology 2010;254:145-53.

16. Pontana F, Duhamel A, Pagniez J, et al. Chest computed tomography using iterative reconstruction vs filtered back projection (part 2): image quality of low-dose CT examinations in 80 patients. Eur Radiol 2011;21:636-43.

17. Pontana F, Pagniez J, Flohr T, et al. Chest computed tomography using iterative reconstruction vs filtered back projection (part 1): evaluation of image noise reduction in 32 patients. Eur Radiol 2011;21:627-35.

18. Ren Q, Dewan SK, Li M, et al. Comparison of adaptive statistical iterative and filtered back projection reconstruction techniques in brain CT. Eur J Radiol 2012;81:2597-601.

19. Silva AC, Lawder HJ, Hara A, et al. Innovations in CT dose reduction strategy: application of the adaptive statistical iterative reconstruction algorithm. AJR Am J Roentgenol 2010;194:191-9.
20. Singh S, Kalra MK, Gilman MD, et al. Adaptive statistical iterative reconstruction technique for radiation dose reduction in chest $\mathrm{CT}$ : a pilot study. Radiology 2011;259:565-73.

21. Mieville FA, Gudinchet F, Brunelle F, et al. Iterative reconstruction methods in two different MDCT scanners: physical metrics and 4-alternative forced-choice detectability experiments-a phantom approach. Phys Med 2013;29:99-110.

22. Gevenois PA, de Maertelaer V, Madani A, et al. Asbestosis, pleural plaques and diffuse pleural thickening: three distinct benign responses to asbestos exposure. Eur Respir J 1998;11:1021-7.

23. Roach HD, Davies GJ, Attanoos R, et al. Asbestos: when the dust settles an imaging review of asbestos-related disease. Radiographics 2002;22(Spec No):S167-84.

24. Jankowski A, Martinelli T, Timsit JF, et al. Pulmonary nodule detection on MDCT images: evaluation of diagnostic performance using thin axial images, maximum intensity projections, and computer-assisted detection. Eur Radiol 2007;17:3148-56.

25. Deak PD, Smal Y, Kalender WA. Multisection CT protocols: sex- and age-specific conversion factors used to determine effective dose from dose-length product. Radiology 2010;257:158-66.

26. Szucs-Farkas Z, Strautz T, Patak MA, et al. Is body weight the most appropriate criterion to select patients eligible for low-dose pulmonary CT angiography? Analysis of objective and subjective image quality at $80 \mathrm{kVp}$ in 100 patients. Eur Radiol 2009;19:1914-22.

27. Nam JM. Testing the intraclass version of kappa coeffcient of agreement with binary scale and sample size determination. Biom $\mathrm{J}$ 2002;44:558-70.

28. Cicchetti DV, Feinstein AR. High agreement but low kappa: II. Resolving the paradoxes. J Clin Epidemiol 1990;43:551-8.

29. Feinstein AR, Cicchetti DV. High agreement but low kappa: I. The problems of two paradoxes. J Clin Epidemiol 1990;43:543-9.

30. Mieville FA, Berteloot L, Grandjean A, et al. Model-based iterative reconstruction in pediatric chest CT: assessment of image quality in a prospective study of children with cystic fibrosis. Pediatr Radiol 2013;43:558-67.

31. Friedman AC, Fiel SB, Fisher MS, et al. Asbestos-related pleural disease and asbestosis: a comparison of CT and chest radiography. AJR Am J Roentgenol 1988;150:269-75.

32. Ross RM. The clinical diagnosis of asbestosis in this century requires more than a chest radiograph. Chest 2003;124:1120-8.

33. Green FH, Harley R, Vallyathan V, et al. Exposure and mineralogical correlates of pulmonary fibrosis in chrysotile asbestos workers. Occup Environ Med 1997:54:549-59.

34. Paris C, Benichou J, Raffaelli C, et al. Factors associated with early-stage pulmonary fibrosis as determined by high-resolution computed tomography among persons occupationally exposed to asbestos. Scand J Work Environ Health 2004;30:206-14

35. MacMahon $\mathrm{H}$, Austin JH, Gamsu G, et al. Guidelines for management of small pulmonary nodules detected on CT scans: a statement from the Fleischner Society. Radiology 2005;237: 395-400. 